\title{
The Search for Millisecond Pulsars - Implications from Fermi Observations and Future Prospects
}

\author{
Colin J. Clark ${ }^{* \dagger \ddagger}$ \\ Jodrell Bank Centre for Astrophysics, School of Physics and Astronomy, The University of \\ Manchester, Manchester M13 9PL, UK \\ Albert-Einstein-Institut, Max-Planck-Institut für Gravitationsphysik, D-30167 Hannover, \\ Germany \\ Leibniz Universität Hannover, D-30167 Hannover, Germany \\ E-mail: colin.clark-2@manchester.ac.uk
}

\begin{abstract}
Millisecond pulsars (MSPs) are old pulsars which have been spun up to incredible rotation rates by the accretion of matter from an orbiting companion star. Their extreme properties and long-term stability make them valuable objects for a wide variety of fundamental astrophysics. In recent years, the rate of new MSP discoveries has increased dramatically, owing in large part to the "treasure trove" of pulsar-like sources detected within the LAT data. In fact, more than a quarter of all Galactic-field MSPs were discovered in searches targeting unidentified Fermi-LAT sources. In these proceedings I describe the various contributions that Fermi has made to the MSP search effort, including the latest results from blind searches for gamma-ray pulsations from unknown pulsars. I also discuss the prospects for future discoveries in these areas, and the implications of Fermi's observations for the Galactic MSP population.
\end{abstract}

7th Fermi Symposium 2017

15-20 October 2017

Garmisch-Partenkirchen, Germany

\footnotetext{
* Speaker.

${ }^{\dagger}$ on behalf of the Fermi-LAT collaboration

${ }^{\ddagger}$ C.J.C. acknowledges support from the ERC under the European Union’s Horizon 2020 research and innovation programme (grant agreement No. 715051; Spiders). The Fermi-LAT Collaboration acknowledges support for LAT development, operation and data analysis from NASA and DOE (United States), CEA/Irfu and IN2P3/CNRS (France), ASI and INFN (Italy), MEXT, KEK, and JAXA (Japan), and the K.A. Wallenberg Foundation, the Swedish Research Council and the National Space Board (Sweden). Science analysis support in the operations phase from INAF (Italy) and CNES (France) is also gratefully acknowledged. This work performed in part under DOE Contract DE-AC02$76 \mathrm{SF} 00515$.
} 


\section{Introduction}

Millisecond pulsars (MSPs) are old neutron stars that rotate up to several hundred times per second as the result of having been "spun up" (or "recycled") by accreting matter from an orbiting companion star [1]. Since the original discovery of PSR B1937+21, a $1.56 \mathrm{~ms}$ pulsar within a puzzling unidentified radio source [2], around 350 MSPs (with periods below $30 \mathrm{~ms}$ ) have been discovered through their radio pulsations [3] ${ }^{1}$.

The extreme rotational stability of MSPs, in combination with their rapid spin rates make them exceptionally useful astrophysical tools: small changes in the light travel time between the pulsar and an observer are measurable as shifts in the arrival times of pulses, allowing "timing" analyses with MSPs to probe a wide range of dynamical processes. Notable examples include: the discovery of the first extra-solar planets [4]; extremely precise tests of general relativity using the mildy-recycled "double pulsar" system PSR J0737-3039A/B [5]; and even "pulsar timing arrays" (PTAs) attempting to use MSPs to detect a stochastic background of low-frequency gravitational waves by measuring correlated variations in their arrival times [6].

Prior to Fermi's launch, gamma-ray pulsations had only been detected from a handful of young pulsars, and just one MSP [7]. This changed almost immediately, with the Large Area Telescope (LAT) detecting gamma-ray pulsations from eight MSPs [8]. This quickly confirmed that, despite the differences in the physical properties of MSPs and young pulsars (e.g. surface magnetic field strengths more than a thousand times lower), MSPs are also prolific gamma-ray emitters, and have pulse profiles remarkably similar to those of the young gamma-ray detected pulsars [9].

Since then, the LAT has become an indispensable instrument for pulsar astronomy [10]. Of the more than 200 pulsars from which the LAT has detected pulsations (see contribution by L. Guillemot to these proceedings) ${ }^{2}$, almost half are MSPs, and a large number of these in fact owe their discoveries directly to the LAT (see Figure 1).

\section{MSP discoveries in Fermi-LAT sources}

Arguably the most important of Fermi's contributions to pulsar astronomy is the LAT's "treasure trove" of pulsar candidate sources (identified by their curved gamma-ray spectra and low flux variability) which can be targeted for sensitive dedicated searches with radio telescopes $[9,10]$. To fully exploit this, the Pulsar Search Consortium (PSC) was formed [11] to facilitate the sharing of candidate pulsars between the Fermi-LAT collaboration and radio astronomers across the globe. Since Fermi's launch in 2008, these searches have been driving the discoveries of new MSPs, far accelerating their discovery rate over blind radio surveys alone (see Figure 2). PSC searches of unidentified LAT sources $[12,13]$ have now discovered more than 80 new MSPs, almost one-third of all known MSPs in the Galactic field.

Future searches by the PSC are expected to continue to lead future MSP discoveries, as new LAT source catalogues are produced, and as new radio telescopes begin operating. A recent example is the new participation of LOFAR (the LOw Frequency ARray) in the PSC. LOFAR is the first major radio telescope to perform pulsar searches at radio frequencies below $250 \mathrm{MHz}$, allowing

\footnotetext{
${ }^{1}$ http://www.atnf.csiro.au/research/pulsar/psrcat

${ }^{2}$ For a current list of published gamma-ray pulsars, see http://tinyurl.com/fermipulsars
} 


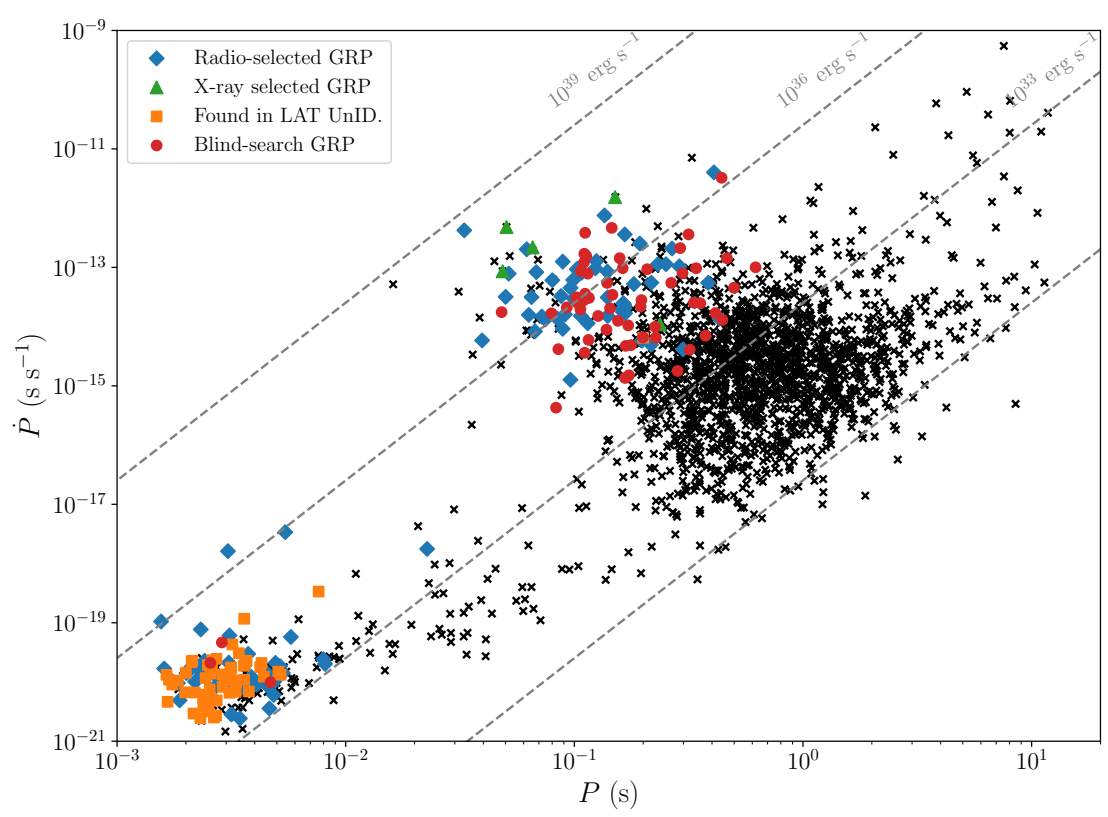

Figure 1: Period-Period-derivative $(P-\dot{P})$ diagram, showing the known radio and X-ray pulsars (black crosses) and gamma-ray pulsars (colored markers). The population in the lower-left corner are "recycled" or millisecond pulsars. Dashed diagonal lines show lines of constant spin-down power $\left(\dot{E}=4 \pi^{2} I \dot{P} / P^{3}\right.$, with an assumed moment of inertia, $I=10^{45} \mathrm{~g} \mathrm{~cm}^{2}$ ). Of particular note is the large number of gamma-ray MSPs discovered in radio searches of unidentified Fermi-LAT sources (orange squares).

it to detect pulsars with steep spectra [14] which may have been missed by more typical L-band $(1.4 \mathrm{GHz})$ surveys. This exciting discovery potential was recently demonstrated by LOFAR's first two MSP discoveries, both within previously unassociated LAT sources [15, 16]. One of these, the binary MSP J0952-0607, is particularly notable as the second fastest spinning pulsar known, with a rotational period of just $1.41 \mathrm{~ms}$.

The near future for the MSP search effort is particularly exciting, with the new Five-hundred Metre Aperture Spherical Telescope (FAST) already beginning to find new pulsars ${ }^{3}$, and the 64dish MeerKAT array ${ }^{4}$ set to become the most powerful radio telescope in the Southern hemisphere when it begins operating next year. Targeted searches of unidentified Fermi-LAT sources are being pursued by both teams. Looking further ahead, the Square Kilometre Array (SKA) [17], of which MeerKAT will form the core, is set to revolutionise pulsar astronomy in the next decades. Consisting of around 200 dishes, and more than 100,000 low frequency antennas across Australia and South Africa, the SKA is set to increase the known pulsar population by an order of magnitude, revealing a large fraction of all pulsars within the Galaxy.

\section{Spider MSPs}

One area in which the LAT's ability to identify pulsar candidates has had particular impact has been the discovery of a large number of "spider" MSPs: binary systems consisting of an MSP and

\footnotetext{
${ }^{3}$ For confirmed FAST pulsar discoveries, see http://crafts.bao.ac.cn/pulsar/fast_all_pulsar_list

${ }^{4}$ http://www.ska.ac.za/science-engineering/meerkat/about-meerkat/
} 


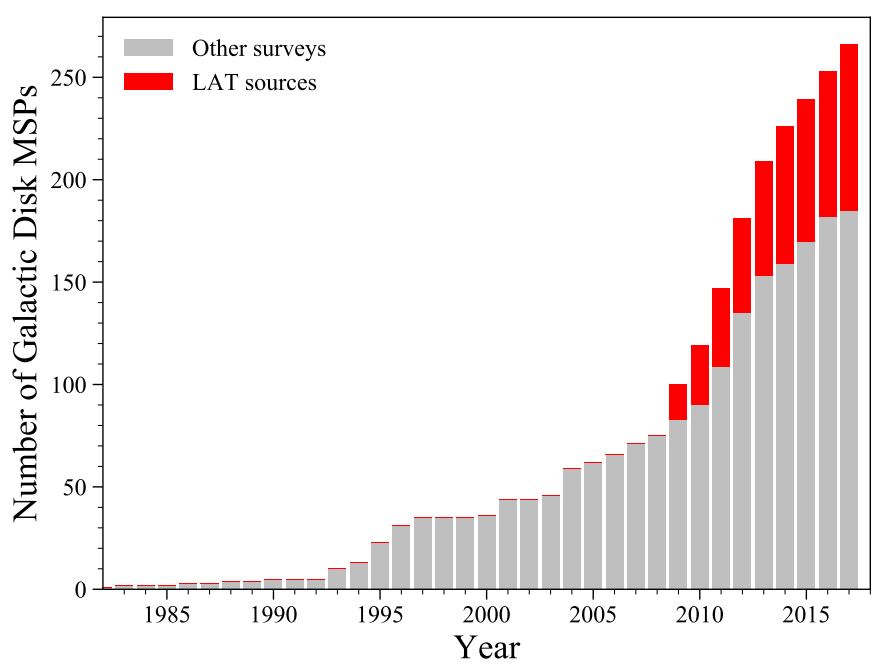

Figure 2: Cumulative number of known MSPs in the Galactic field (i.e. outside globular clusters) as a function of time, taken from the list of MSP discoveries available at http://astro.phys.wvu.edu/GalacticMSPs/. MSPs highlighted in red, accounting for more than a quarter of the total, were discovered in dedicated searches of unidentified Fermi-LAT sources.

a low-mass, semi-degenerate companion star (as opposed to a white dwarf or neutron star companion) which is irradiated by the pulsar's high-energy wind. These systems are further classified into "black widows" or "redbacks" depending on the companion star's mass $\left(M_{c}<0.05 M_{\odot}\right.$ vs $M_{c}<0.2-0.4 M_{\odot}$ respectively). These nicknames come from species of spiders in which the larger females prey upon their mates, reflecting the fact that the pulsars in these systems ablate and may ultimately destroy their lighter companion stars.

This ablation process often leads to a large amount of material being stripped from the companion star into the binary system, which can eclipse the pulsar's radio pulsations [18]. While gamma-ray emission can pass through this material unobstructed, these eclipses make radio pulsations from spider MSPs undetectable for large fractions of their orbits. Repeated searches are therefore often required to detect these objects outside of their eclipses, and such searches are only feasible when a promising pulsar candidate location has been identified by the LAT, or towards globular clusters. As a result, searches targeting LAT sources have increased the number of known spiders in the Galactic field by more than a factor of 5, from just 4 before Fermi's launch to more than 25 now.

These systems are at a crucial stage of the recycling process, close to or just beyond the point at which the system evolves from an accreting low-mass X-ray binary (LMXB) into a rotationallypowered MSP binary. Indeed, the LAT has observed this metamorphosis in real-time in the form of so-called "transitional MSPs", systems observed to switch between LMXB and MSP states on timescales of a few years [19]. Three such objects are currently known, and the LAT has observed striking changes in the gamma-ray flux from two of these [20,21], including gamma-ray pulsations from one in its MSP state. At least two other LAT sources (3FGL J1544.6-1125 [22] and 3FGL J0427.9-6704 [23]) have been identified as potential transitional MSPs, raising hopes that more transitions may be observed in future LAT data. 
One particularly interesting example of the LAT's observations helping to overcome the difficulties in discovering and studying eclipsing spider MSPs, is the story of the discovery and subsequent timing of the redback PSR J2339-0533. Like many other MSPs, this pulsar was first identified as a promising gamma-ray pulsar candidate by its significantly curved gamma-ray spectrum and low flux variability. A search for new spider systems in optical and X-ray data discovered a periodically variable counterpart [24] (caused by the pulsar heating one side of the companion star), all but confirming the spider identification until the eventual discovery of its radio pulsations [25]. However, when the radio ephemeris was applied to the LAT data, gamma-ray pulsations were only detected over a short portion of the LAT data. A timing analysis using the gamma-ray data itself was required to resolve this, and revealed the source of the problem: the pulsar's orbital period was varying significantly and non-monotonically over time [26]. One possible explanation for this is that the companion star's gravitational quadrupole moment is changing, i.e. it becomes more or less oblate over time, and the resulting rotational angular momentum changes couple with the orbital angular momentum of the system, leading to the observed orbital period variations. These effects are observed in several other redback systems; gamma-ray timing analyses using the LAT data will be able to obtain phase-connected timing solutions covering the LAT observation span without being affected by the orbital eclipses which otherwise limit long-term radio timing campaigns (see contribution by L. Nieder to these proceedings).

The discovery route of PSR J2339-0533 is a journey down which a number of other systems have only just begun: these are unidentified, pulsar-like gamma-ray sources within which a periodically variable optical counterpart has been discovered, but from which no pulsations have been detected, despite repeated radio searches ${ }^{5}$. These "missing spiders" (see contribution by A. Kong to these proceedings) contain some unique systems, including the spider candidate with the shortest known orbital period, 3FGL J1653.6-0158 [27, 28] and an unusual, apparently eccentric system 3FGL J0523.3-2528 [29]. The lack of radio pulsations from these systems is likely to be due to extreme eclipsing effects, but could alternatively be explained by unfortunate viewing/beaming angles causing their radio emission beams to miss the line-of-sight to Earth. One promising avenue to detect pulsations from these systems, despite their apparent radio-quietness, is to perform blind searches for pulsations within the LAT data.

\section{Blind gamma-ray pulsation searches}

Blindly searching for gamma-ray pulsations from an unknown pulsar using Fermi-LAT data is an extremely computationally expensive task. Due to the sparseness of the gamma-ray photon arrival times long integrations over months or years of data are required, meaning the signal parameters (spin frequency, spin-down rate, sky position and orbital parameters) must be searched with extremely high resolution. In fact, performing a fully coherent search, consistently accounting for every rotation of the pulsar over the entire integration, requires many more search trials than can feasibly be performed, even with the fastest modern supercomputers.

\footnotetext{
${ }^{5}$ The author proposes that such systems be called "recluse" spiders, in keeping with the theme of naming irradiated binary systems after poisonous spider species, but reflecting the fact that these do not seem to want to reveal themselves to us!
} 
To get around this problem, efficient "time differencing" or "semicoherent" techniques were developed prior to the beginning of the LAT mission [30], in which photon arrival times were only accounted for coherently if they arrived within a short time window of one another. This reduces the required parameter space resolution dramatically, while still maintaining sensitivity to pulsations. Soon after Fermi's launch, blind searches of LAT data discovered sixteen new gamma-ray pulsars [31], with ten more following over the first two years of the mission [32].

However, all of these pulsars were young gamma-ray pulsars. The blind-search discovery of an MSP is even more challenging: the higher spin frequencies require even higher precision when performing position-dependent barycentering corrections to the photon arrival times, meaning that a far denser grid of sky locations must be searched in order to detect an MSP. Using methods derived from searches for continuous gravitational waves to account for this, a new wave of blind searches quickly discovered another ten young pulsars [33].

The most spectacular blind-search discovery, however, was the detection of a new black widow, PSR J1311-3430. Much like PSR J2339-0533 (above), this was originally identified as a pulsar-like gamma-ray source with a periodically variable optical counterpart [34]. Constraints on the orbital parameters from the counterpart's optical lightcurve were then used to perform a targeted pulsation search, resulting in the first and so-far only blind-search binary MSP [35]. This discovery leaves us optimistic that the identification of the missing spiders (above) may soon be possible with improved search methods and orbital ephemerides (see L. Nieder, these proceedings).

As the LAT data set grows, and as searches attempt to detect fainter pulsars, the computational cost of blind searches continues to increase. To meet the growing demands, the blind search effort was moved to the Einstein@Home project, which distributes computations amongst tens of thousands of volunteers' computers. Participants in the Einstein@ Home project have now discovered 23 gamma-ray pulsars [36, 37, J. Wu, et al. 2017, submitted]. This number includes two new isolated MSPs [Clark et al. 2017, submitted], discoveries which confirm the sensitivity of blind gamma-ray pulsation searches to a so-far unseen population of radio-quiet MSPs. Following on from this, Einstein@Home is currently performing GPU-accelerated searches for binary gammaray MSPs, with the aim of detecting gamma-ray pulsations from promising spider candidates.

\section{Implications and future prospects}

Recently, a new way to discover pulsars amongst the Fermi-LAT treasure trove has emerged. As mentioned above, MSPs appear to have steep radio spectra, but searches are often hampered by the increased effects of scattering and dispersion at low frequencies [16]. The new method circumvents these issues by instead searching for the time-averaged emission from pulsars in lowfrequency radio images (indeed the original MSP was discovered within a low-frequency continuum source [2]). By cross-correlating sources from the $150 \mathrm{MHz}$ GMRT Sky Survey with higher-frequency surveys, a number of steep spectrum sources were identified [38]. Further crosscorrelation with Fermi-LAT source catalogues revealed a small number of steep-spectrum pulsar candidates within the localization regions of unidentified gamma-ray sources. The discovery rate from these has been exceptional: out of just 16 candidates within 3FGL and preliminary 8-year catalogue sources, 6 new MSPs (including 3 black widows) have been discovered [Frail et al. 2017, submitted, Clark et al. 2017, in preparation]. As new Fermi-LAT source catalogues are produced, 
and new sky surveys performed (e.g. by LOFAR), this new search technique has the potential to drive future gamma-ray MSP discoveries and address the significant biases against finding steep spectrum MSPs.

Finally, perhaps one of the most exciting surprises of the Fermi-LAT observations is the discovery of an extended excess of $\mathrm{GeV}$ emission towards the inner galaxy (see contribution of D. Malyshev to these proceedings for an overview). Several recent studies have shown that this excess is consistent with an unresolved population of point sources [39, 40]. Based on the spectrum of this excess, and its spatial extension, MSPs are a leading candidate constituent, with analyses suggesting a so-far unseen population of thousands of MSPs within the Galactic bulge [41]. However, the large degree of scattering and dispersion in the inner-galaxy prevents radio searches from detecting pulsars in this region, at least with current technology. Future pulsar searches with MeerKAT (and eventually SKA) targeting Fermi-LAT sources in the inner galaxy may be able to reveal this predicted population. Until then, blind gamma-ray pulsation searches may be able to discover the brightest members of this population; Einstein@Home is currently pursuing this goal by searching for pulsations from unidentified sources from "2FIG" inner-galaxy sources [42]. In either case, if this population does exist, Fermi will be instrumental in its discovery.

\section{References}

[1] M. A. Alpar, A. F. Cheng, M. A. Ruderman and J. Shaham, A new class of radio pulsars, Nature 300 (Dec., 1982) 728-730.

[2] D. C. Backer, S. R. Kulkarni, C. Heiles, M. M. Davis and W. M. Goss, A millisecond pulsar, Nature 300 (Dec., 1982) 615-618.

[3] R. N. Manchester, G. B. Hobbs, A. Teoh and M. Hobbs, The Australia Telescope National Facility Pulsar Catalogue, AJ 129 (Apr., 2005) 1993-2006, [astro-ph/ 0412641 ].

[4] A. Wolszczan and D. A. Frail, A planetary system around the millisecond pulsar PSR1257 + 12, Nature 355 (Jan., 1992) 145-147.

[5] M. Kramer, I. H. Stairs, R. N. Manchester, M. A. McLaughlin, A. G. Lyne, R. D. Ferdman et al., Tests of General Relativity from Timing the Double Pulsar, Science 314 (Oct., 2006) 97-102, [astro-ph/0609417].

[6] J. P. W. Verbiest, L. Lentati, G. Hobbs, R. van Haasteren, P. B. Demorest et al., The International Pulsar Timing Array: First data release, MNRAS 458 (May, 2016) 1267-1288, [1602.03640].

[7] L. Kuiper, W. Hermsen, F. Verbunt, D. J. Thompson, I. H. Stairs, A. G. Lyne et al., The likely detection of pulsed high-energy gamma -ray emission from millisecond pulsar PSR J0218+4232, A\&A 359 (July, 2000) 615-626, [astro-ph/ 0005338 ].

[8] A. A. Abdo, M. Ackermann, M. Ajello, W. B. Atwood, M. Axelsson et al., A Population of Gamma-Ray Millisecond Pulsars Seen with the Fermi Large Area Telescope, Science 325 (Aug., 2009) 848.

[9] I. A. Grenier and A. K. Harding, Gamma-ray pulsars: A gold mine, Comptes Rendus Physique 16 (Aug., 2015) 641-660, [1509.08823].

[10] P. A. Caraveo, Gamma-Ray Pulsar Revolution, ARA\&A 52 (Aug., 2014) 211-250, [1312.2913]. 
[11] P. S. Ray et al., Radio Searches of Fermi LAT Sources and Blind Search Pulsars: The Fermi Pulsar Search Consortium, arXiv:1205.3089 (2012) .

[12] F. Camilo, M. Kerr, P. S. Ray, S. M. Ransom, J. Sarkissian et al., Parkes Radio Searches of Fermi Gamma-Ray Sources and Millisecond Pulsar Discoveries, ApJ 810 (Sept., 2015) 85, [1507. 04451 ].

[13] H. T. Cromartie, F. Camilo, M. Kerr, J. S. Deneva, S. M. Ransom, P. S. Ray et al., Six New Millisecond Pulsars from Arecibo Searches of Fermi Gamma-Ray Sources, ApJ 819 (Mar., 2016) 34, [1601.05343].

[14] D. A. Frail, P. Jagannathan, K. P. Mooley and H. T. Intema, Known Pulsars Identified in the GMRT $150 \mathrm{MHz}$ All-Sky Survey, ArXiv e-prints (June, 2016), [1606. 004 4 9].

[15] Z. Pleunis, C. G. Bassa, J. W. T. Hessels, V. I. Kondratiev, F. Camilo, I. Cognard et al., A Millisecond Pulsar Discovery in a Survey of Unidentified Fermi $\gamma$-Ray Sources with LOFAR, ApJ 846 (Sept., 2017) L19, [1709.01452].

[16] C. G. Bassa, Z. Pleunis, J. W. T. Hessels, E. C. Ferrara, R. P. Breton, N. V. Gusinskaia et al., LOFAR Discovery of the Fastest-spinning Millisecond Pulsar in the Galactic Field, ApJ 846 (Sept., 2017) L20, [1709.01453].

[17] M. Kramer and B. Stappers, Pulsar Science with the SKA, Advancing Astrophysics with the Square Kilometre Array (AASKA14) (Apr., 2015) 36, [1507.04423].

[18] J. S. Deneva, P. S. Ray, F. Camilo, J. P. Halpern, K. Wood, H. T. Cromartie et al., Multiwavelength Observations of the Redback Millisecond Pulsar J1048+2339, ApJ 823 (June, 2016) 105, [1601.03681].

[19] A. M. Archibald, I. H. Stairs, S. M. Ransom, V. M. Kaspi, V. I. Kondratiev, D. R. Lorimer et al., A Radio Pulsar/X-ray Binary Link, Science 324 (June, 2009) 1411, [0 905.3397 ].

[20] B. W. Stappers, A. M. Archibald, J. W. T. Hessels, C. G. Bassa, S. Bogdanov, G. H. Janssen et al., A State Change in the Missing Link Binary Pulsar System PSR J1023+0038, ApJ 790 (July, 2014) 39, [1311. 7506].

[21] T. J. Johnson, P. S. Ray, J. Roy, C. C. Cheung, A. K. Harding, H. J. Pletsch et al., Discovery of Gamma-Ray Pulsations from the Transitional Redback PSR J1227-4853, ApJ 806 (June, 2015) 91, [1502.06862].

[22] S. Bogdanov and J. P. Halpern, Identification of the High-energy Gamma-Ray Source 3FGL J1544.6-1125 as a Transitional Millisecond Pulsar Binary in an Accreting State, ApJ 803 (Apr., 2015) L27, [1503.01698].

[23] J. Strader, K.-L. Li, L. Chomiuk, C. O. Heinke, A. Udalski, M. Peacock et al., A New $\gamma$-Ray Loud, Eclipsing Low-mass X-Ray Binary, ApJ 831 (Nov., 2016) 89, [1608. 02583$].$

[24] R. W. Romani and M. S. Shaw, The Orbit and Companion of Probable $\gamma$-Ray Pulsar J2339-0533, ApJ 743 (Dec., 2011) L26, [1111. 3074$].$

[25] P. S. Ray, A. M. Belfiore, P. Saz Parkinson, E. Polisensky, S. M. Ransom, R. W. Romani et al., Discovery of the radio and gamma-ray pulsar PSR J2339-0533 associated with the Fermi LAT bright source OFGL J2339.8-0530, in American Astronomical Society Meeting Abstracts \#223, vol. 223 of American Astronomical Society Meeting Abstracts, p. 140.07, Jan., 2014.

[26] H. J. Pletsch and C. J. Clark, Gamma-Ray Timing of Redback PSR J2339-0533: Hints for Gravitational Quadrupole Moment Changes, ApJ 807 (July, 2015) 18, [1504.07466]. 
[27] A. K. H. Kong, R. Jin, T.-C. Yen, C.-P. Hu, C. Y. Hui, P. H. T. Tam et al., Discovery of an Ultracompact Gamma-Ray Millisecond Pulsar Binary Candidate, ApJ 794 (Oct., 2014) L22, [1408.5162].

[28] R. W. Romani, A. V. Filippenko and S. B. Cenko, 2FGL J1653.6-0159: A New Low in Evaporating Pulsar Binary Periods, ApJ 793 (Sept., 2014) L20, [14 08 . 2886].

[29] J. Strader, L. Chomiuk, E. Sonbas, K. Sokolovsky, D. J. Sand, A. S. Moskvitin et al., 1FGL J0523.5-2529: A New Probable Gamma-Ray Pulsar Binary, ApJ 788 (June, 2014) L27, [1405.5533].

[30] W. B. Atwood, M. Ziegler, R. P. Johnson and B. M. Baughman, A Time-differencing Technique for Detecting Radio-quiet Gamma-Ray Pulsars, ApJ 652 (Nov., 2006) L49-L52.

[31] A. A. Abdo, M. Ackermann, M. Ajello, B. Anderson, W. B. Atwood, M. Axelsson et al., Detection of 16 Gamma-Ray Pulsars Through Blind Frequency Searches Using the Fermi LAT, Science 325 (Aug., 2009) 840, [1009.0748].

[32] P. M. Saz Parkinson, M. Dormody, M. Ziegler, P. S. Ray et al., Eight $\gamma$-ray Pulsars Discovered in Blind Frequency Searches of Fermi LAT Data, ApJ 725 (Dec., 2010) 571-584, [1 006 . 2134 ].

[33] H. J. Pletsch, L. Guillemot, B. Allen, M. Kramer, C. Aulbert, H. Fehrmann et al., Discovery of Nine Gamma-Ray Pulsars in Fermi Large Area Telescope Data Using a New Blind Search Method, ApJ 744 (Jan., 2012) 105, [1111.0523].

[34] R. W. Romani, 2FGL J1311.7-3429 Joins the Black Widow Club, ApJ 754 (Aug., 2012) L25, [1207.1736].

[35] H. J. Pletsch, L. Guillemot, H. Fehrmann, B. Allen, M. Kramer et al., Binary Millisecond Pulsar Discovery via Gamma-Ray Pulsations, Science 338 (Dec., 2012) 1314, [1211.1385].

[36] H. J. Pletsch, L. Guillemot, B. Allen, D. Anderson, C. Aulbert, O. Bock et al., Einstein@Home Discovery of Four Young Gamma-Ray Pulsars in Fermi LAT Data, ApJ 779 (Dec., 2013) L11, [1311.6427].

[37] C. J. Clark, J. Wu, H. J. Pletsch, L. Guillemot, B. Allen, C. Aulbert et al., The Einstein@Home Gamma-ray Pulsar Survey. I. Search Methods, Sensitivity, and Discovery of New Young Gamma-Ray Pulsars, ApJ 834 (Jan., 2017) 106, [1611.01015].

[38] D. A. Frail, K. P. Mooley, P. Jagannathan and H. T. Intema, Pulsar candidates towards Fermi unassociated sources, MNRAS 461 (Sept., 2016) 1062-1067, [1606.03450].

[39] S. K. Lee, M. Lisanti, B. R. Safdi, T. R. Slatyer and W. Xue, Evidence for Unresolved $\gamma$-Ray Point Sources in the Inner Galaxy, Physical Review Letters 116 (Feb., 2016) 051103, [1506. 05124 ].

[40] R. Bartels, S. Krishnamurthy and C. Weniger, Strong Support for the Millisecond Pulsar Origin of the Galactic Center GeV Excess, Physical Review Letters 116 (Feb., 2016) 051102, [1506. 05104 ].

[41] F. Calore, M. Di Mauro, F. Donato, J. W. T. Hessels and C. Weniger, Radio Detection Prospects for a Bulge Population of Millisecond Pulsars as Suggested by Fermi-LAT Observations of the Inner Galaxy, ApJ 827 (Aug., 2016) 143, [1512.06825].

[42] Fermi-LAT Collaboration, Characterizing the population of pulsars in the Galactic bulge with the Fermi Large Area Telescope, ArXiv e-prints (Apr., 2017), [1705. 0000 9]. 